\title{
The extended Baryon Oscillation Spectroscopic Survey: Variability selection and quasar luminosity function (Corrigendum)
}

N. Palanque-Delabrouille ${ }^{1}$, Ch. Magneville ${ }^{1}$, Ch. Yèche ${ }^{1}$, I. Pâris ${ }^{2}$, P. Petitjean ${ }^{3}$, E. Burtin ${ }^{1}$, K. Dawson ${ }^{4}$, I. McGreer ${ }^{5}$, A. D. Myers ${ }^{6}$, G. Rossi ${ }^{7}$, D. Schlegel ${ }^{8}$, D. Schneider ${ }^{9,10}$, A. Streblyanska ${ }^{11,12}$, and J. Tinker ${ }^{13}$

1 CEA, Centre de Saclay, Irfu/SPP, 91191 Gif-sur-Yvette, France e-mail: nathalie.palanque-delabrouille@cea.fr

2 INAF-Osservatorio Astronomico di Trieste, via G. B. Tiepolo 11, 34131 Trieste, Italy

3 UPMC-CNRS, UMR7095, Institut d'Astrophysique de Paris, 75014 Paris, France

${ }^{4}$ Department of Physics and Astronomy, University of Utah, Salt Lake City, UT 84112, USA

5 Steward Observatory, University of Arizona, 933 North Cherry Avenue, Tucson, AZ 85 721, USA

6 Department of Physics and Astronomy, University of Wyoming, Laramie, WY 82 071, USA

7 Department of Astronomy and Space Science, Sejong University, 143-747 Seoul, Korea

${ }^{8}$ Lawrence Berkeley National Laboratory, 1 Cyclotron Road, Berkeley, CA 94 720, USA

9 Department of Astronomy and Astrophysics, 525 Davey Laboratory, The Pennsylvania State University, University Park, PA 16802 , USA

10 Institute for Gravitation and the Cosmos, The Pennsylvania State University, University Park, PA 16802, USA

11 Instituto de Astrofisica de Canarias (IAC), 38200 La Laguna, Tenerife, Spain

12 Universidad de La Laguna (ULL), Dept. Astrofisica, 38206 La Laguna, Tenerife, Spain

13 Center for Cosmology and Particle Physics, New York University, New York, NY 10 003, USA

A\&A 587, A41 (2016), DOI: 10.1051/0004-6361/201527392

Key words. quasars: general - large-scale structure of Universe - surveys - errata, addenda

An error occurred in Tables 6 and 7 during the production process.

The correct tables are published below. 
Table 6. Predicted differential quasar counts over $15.5<g<25$ and $0<z<6$ for a survey covering 10000 deg $^{2}$, based on our best-fit PLE or PLE+LEDE luminosity function model.

\begin{tabular}{|c|c|c|c|c|c|c|c|}
\hline \multicolumn{8}{|c|}{ PLE model } \\
\hline$g \backslash z$ & 0.5 & 1.5 & 2.5 & 3.5 & 4.5 & 5.5 & Total \\
\hline 15.75 & 26 & 2 & 10 & 0 & 0 & 0 & 38 \\
\hline 16.25 & 58 & 8 & 33 & 0 & 0 & 0 & 100 \\
\hline 16.75 & 143 & 39 & 87 & 3 & 0 & 0 & 272 \\
\hline 17.25 & 386 & 180 & 231 & 10 & 0 & 0 & 806 \\
\hline 17.75 & 1134 & 813 & 626 & 26 & 0 & 0 & 2599 \\
\hline 18.25 & 3396 & 3468 & 1727 & 68 & 0 & 0 & 8660 \\
\hline 18.75 & 8930 & 12525 & 4780 & 179 & 0 & 0 & 26413 \\
\hline 19.25 & 17963 & 32977 & 12319 & 462 & 1 & 0 & 63722 \\
\hline 19.75 & 28172 & 61064 & 26357 & 1170 & 3 & 0 & 116766 \\
\hline 20.25 & 38732 & 90121 & 45742 & 2827 & 7 & 0 & 177430 \\
\hline 20.75 & 50756 & 121102 & 68898 & 6299 & 18 & 0 & 247074 \\
\hline 21.25 & 65550 & 157999 & 95351 & 12540 & 47 & 0 & 331487 \\
\hline 21.75 & 84300 & 204485 & 125298 & 22098 & 120 & 0 & 436302 \\
\hline 22.25 & 108276 & 264163 & 159894 & 35046 & 301 & 1 & 567680 \\
\hline 22.75 & 138990 & 341219 & 201144 & 51351 & 737 & 3 & 733442 \\
\hline 23.25 & 178321 & 440913 & 251597 & 70978 & 1722 & 7 & 943538 \\
\hline 23.75 & 228612 & 570015 & 314273 & 93793 & 3739 & 17 & 1210449 \\
\hline 24.25 & 292750 & 737314 & 392821 & 119723 & 7357 & 42 & 1550006 \\
\hline 24.75 & 374172 & 954240 & 491772 & 149132 & 13010 & 106 & 1982433 \\
\hline Total & 1620667 & 3992648 & 2192958 & 565706 & 27062 & 175 & 8399216 \\
\hline \multicolumn{8}{|c|}{ PLE+LEDE model } \\
\hline$g \backslash z$ & 0.5 & 1.5 & 2.5 & 3.5 & 4.5 & 5.5 & Total \\
\hline 15.75 & 35 & 5 & 1 & 0 & 0 & 0 & 42 \\
\hline 16.25 & 82 & 20 & 5 & 0 & 0 & 0 & 108 \\
\hline 16.75 & 200 & 77 & 20 & 1 & 0 & 0 & 298 \\
\hline 17.25 & 519 & 291 & 76 & 3 & 0 & 0 & 889 \\
\hline 17.75 & 1402 & 1088 & 287 & 10 & 0 & 0 & 2787 \\
\hline 18.25 & 3758 & 3887 & 1065 & 35 & 0 & 0 & 8745 \\
\hline 18.75 & 9092 & 12345 & 3737 & 117 & 0 & 0 & 25291 \\
\hline 19.25 & 17972 & 31289 & 11415 & 382 & 1 & 0 & 61059 \\
\hline 19.75 & 28629 & 59594 & 27445 & 1163 & 4 & 0 & 116836 \\
\hline 20.25 & 39554 & 90405 & 50511 & 3077 & 13 & 0 & 183560 \\
\hline 20.75 & 51163 & 121609 & 76197 & 6648 & 36 & 1 & 255653 \\
\hline 21.25 & 64559 & 155923 & 103193 & 11780 & 97 & 2 & 335554 \\
\hline 21.75 & 80771 & 196666 & 133441 & 18077 & 242 & 4 & 429201 \\
\hline 22.25 & 100758 & 246795 & 169698 & 25349 & 543 & 9 & 543153 \\
\hline 22.75 & 125546 & 309293 & 214695 & 33716 & 1063 & 21 & 684334 \\
\hline 23.25 & 156321 & 387578 & 271321 & 43594 & 1820 & 46 & 860680 \\
\hline 23.75 & 194486 & 485819 & 342970 & 55625 & 2799 & 93 & 1081792 \\
\hline 24.25 & 241694 & 609219 & 433845 & 70598 & 3989 & 173 & 1359518 \\
\hline 24.75 & 299797 & 764319 & 549267 & 89454 & 5393 & 291 & 1708521 \\
\hline Total & 1416338 & 3476223 & 2389189 & 359629 & 16003 & 640 & 7658021 \\
\hline
\end{tabular}

Notes. Bins are centered on the indicated magnitude and redshift values. The ranges in each bin are $\Delta g=0.5$ and $\Delta z=1$. 
N. Palanque-Delabrouille et al.: Quasar luminosity function

Table 7. Predicted differential quasar counts over $15.5<r<24$ and $0<z<6$ for a survey covering 10000 deg$^{2}$, based on our best-fit PLE or PLE+LEDE luminosity function model.

\begin{tabular}{|c|c|c|c|c|c|c|c|}
\hline \multicolumn{8}{|c|}{ PLE model } \\
\hline$r \backslash z$ & 0.5 & 1.5 & 2.5 & 3.5 & 4.5 & 5.5 & Total \\
\hline 15.75 & 54 & 5 & 14 & 0 & 0 & 0 & 73 \\
\hline 16.25 & 127 & 23 & 41 & 3 & 0 & 0 & 194 \\
\hline 16.75 & 323 & 104 & 108 & 9 & 0 & 0 & 544 \\
\hline 17.25 & 860 & 469 & 290 & 25 & 1 & 0 & 1644 \\
\hline 17.75 & 2316 & 2009 & 793 & 64 & 2 & 0 & 5185 \\
\hline 18.25 & 5854 & 7439 & 2204 & 167 & 5 & 0 & 15669 \\
\hline 18.75 & 12619 & 21145 & 5994 & 432 & 12 & 1 & 40203 \\
\hline 19.25 & 22090 & 44412 & 14675 & 1100 & 31 & 2 & 82311 \\
\hline 19.75 & 32538 & 72781 & 29834 & 2700 & 80 & 5 & 137938 \\
\hline 20.25 & 43787 & 102680 & 50203 & 6208 & 201 & 12 & 203090 \\
\hline 20.75 & 57009 & 136093 & 74188 & 12952 & 502 & 30 & 280774 \\
\hline 21.25 & 73477 & 176857 & 101326 & 24039 & 1217 & 75 & 376990 \\
\hline 21.75 & 94414 & 228620 & 132051 & 39635 & 2811 & 189 & 497720 \\
\hline 22.25 & 121186 & 295216 & 167766 & 58919 & 6038 & 470 & 649595 \\
\hline 22.75 & 155438 & 381240 & 210616 & 80786 & 11825 & 1144 & 841048 \\
\hline 23.25 & 199196 & 492527 & 263236 & 104700 & 20944 & 2660 & 1083262 \\
\hline 23.75 & 254945 & 636610 & 328743 & 131042 & 33694 & 5769 & 1390804 \\
\hline Total & 1076235 & 2598227 & 1382081 & 462783 & 77360 & 10356 & 5607043 \\
\hline \multicolumn{8}{|c|}{ PLE+LEDE model } \\
\hline$r \backslash z$ & 0.5 & 1.5 & 2.5 & 3.5 & 4.5 & 5.5 & Total \\
\hline 15.75 & 74 & 12 & 2 & 0 & 0 & 0 & 88 \\
\hline 16.25 & 173 & 46 & 8 & 1 & 0 & 0 & 227 \\
\hline 16.75 & 425 & 173 & 29 & 3 & 0 & 0 & 630 \\
\hline 17.25 & 1063 & 645 & 109 & 11 & 1 & 0 & 1830 \\
\hline 17.75 & 2637 & 2318 & 407 & 37 & 3 & 1 & 5404 \\
\hline 18.25 & 6171 & 7534 & 1476 & 119 & 10 & 3 & 15314 \\
\hline 18.75 & 12760 & 20293 & 4934 & 380 & 27 & 7 & 38401 \\
\hline 19.25 & 22256 & 42853 & 14012 & 1146 & 71 & 15 & 80354 \\
\hline 19.75 & 33079 & 72019 & 31432 & 3068 & 182 & 33 & 139813 \\
\hline 20.25 & 44377 & 102993 & 55189 & 6846 & 432 & 69 & 209907 \\
\hline 20.75 & 56787 & 135480 & 81153 & 12487 & 915 & 133 & 286955 \\
\hline 21.25 & 71385 & 172381 & 108661 & 19287 & 1689 & 232 & 373634 \\
\hline 21.75 & 89178 & 216865 & 139912 & 26781 & 2731 & 367 & 475834 \\
\hline 22.25 & 111154 & 271898 & 177682 & 35182 & 3976 & 534 & 600426 \\
\hline 22.75 & 138390 & 340627 & 224720 & 45094 & 5393 & 732 & 754956 \\
\hline 23.25 & 172133 & 426753 & 283998 & 57240 & 7015 & 962 & 948101 \\
\hline 23.75 & 213829 & 534834 & 359048 & 72418 & 8921 & 1234 & 1190284 \\
\hline Total & 975871 & 2347722 & 1482773 & 280101 & 31368 & 4322 & 5122156 \\
\hline
\end{tabular}

Notes. Bins are centered on the indicated magnitude and redshift values. The ranges in each bin are $\Delta g=0.5$ and $\Delta z=1$. 